\title{
TIC, desarrollo y jóvenes. Un estado de la cuestión
}

\section{ICT, Development and Young People. A State of the Art}

\author{
Padilla de la Torre, M. R. y Medina Mayagoitia, N. I. ${ }^{1}$ \\ Recibido: 23-03-2018 - Aceptado: 14-06-2018 \\ DOI: https://doi.org/10.26441/RC17.2-2018-A15
}

RESUMEN: El presente artículo es una revisión internacional o estado de la cuestión que analizó más de setenta textos de estudios e intervenciones de tecnologías para la información y la comunicación para el desarrollo y el cambio social con el objetivo de identificar sus tendencias, y de manera particular los que hayan tenido como propósito específico el desarrollo de los jóvenes.

Se dan a conocer los resultados de esta sistematización, en la cual destacan las discusiones en torno al concepto de desarrollo, el predominio de trabajos orientados hacia comunidades vulnerables, y la necesidad de orientar este campo hacia usuarios y problemas situados, como es el caso de los jóvenes, un tema que ha sido poco atendido.

Palabras clave: tecnologías de información y comunicación para el desarrollo y el cambio social; desarrollos juveniles; jóvenes.

ABSTRACT: The present article is an international review or state of the art that analyzed more than seventy texts of studies and interventions of information and communication technologies for development and social change with the objective of identifying tendencies, in particular those that had the specific purpose of youth development.

In the results of this systematization stands out the discussions around the concept of development, the predominance of works in marginalized communities, and the need to orient this field towards situated users and problems, as is the case of young people, a topic that has been less attended.

Keywords: information and communication technologies for development and social change; youth development; young people.

1 María Rebeca Padilla de la Torre es Doctora en Estudios Científico-Sociales por el Instituto Tecnológico de Estudios Superiores de Occidente (ITECO) y Master en Comunicación por la Universidad de Guadalajara. Se desempeña como Profesora-Investigadora del Departamento de Comunicación de la Universidad Autónoma de Aguascalientes (México). rebecapadilla.uaa@gmail.com, http://orcid.org/0000-0002-5881-3958

Norma Isabel Medina Mayagoitia es Maestra en Educación y Profesora-Investigadora del Departamento de Comunicación en el Centro de Ciencias Sociales y Humanidades de la Universidad Autónoma de Aguascalientes. nimedina@correo.uaa.mx 


\section{Introducción. Tecnologías de la Información y la Comunicación para el Desarrollo}

A partir de la década de los ochenta, del siglo pasado, se comenzó a perfilar el papel clave de las tecnologías de la información y comunicación (TIC) para mejorar el bienestar de la población, por ello en las cumbres mundiales sobre la Sociedad de la Información se ha trabajado en alinear sus acciones para lograr los objetivos del milenio y actualmente los objetivos de desarrollo sostenible World Summit on the Information Society (WSIS, 2017). Sin embargo, el principal obstáculo ha sido la desigual distribución y falta de acceso a las TIC, fundamentalmente entre población vulnerable y marginada. Con el fin de contrarrestar lo anterior, se han llevado a cabo varios esfuerzos en el diseño e implementación de políticas públicas; no obstante, éstos no han sido suficientes.

Este trabajo ${ }^{2}$ presenta un estado de la cuestión sobre las tendencias internacionales en el campo académico de la línea de TIC para el desarrollo, Information and Communication Technologies for Development (ICT4D), dentro de la cual precisamente se cuestiona por qué dichos esfuerzos sostenidos para promover el desarrollo de la población a través de las TIC no han tenido los resultados esperados.

La discusión al respecto es que esto se debe a que el concepto de desarrollo se ha basado en el paradigma de la modernidad. Baelden \& Audenhove
(2015) argumentan que en la promoción del desarrollo por medio de las TIC ha faltado una comprensión de las necesidades y perspectivas de los propios usuarios, insertos en las lógicas de sus contextos particulares. En este marco, se inserta la hipótesis que orienta esta revisión: los estudios e intervenciones de TIC para el desarrollo, en general, no tienen el impacto esperado porque no atienden el contexto y la visión de los usuarios de manera más específica, como es el caso de los jóvenes.

Una definición amplia de ICT4D señala que son "tecnologías de comunicación individuales o en grupo cuya adopción o impacto constituyen soportes para el logro de objetivos y aspiraciones de desarrollo actual o futuro" (Heffernan, Lin \& Thomson, 2016, p. 4). Por su parte, Johri \& Pal (2012) explican que en la pasada década han sido muy significativos los trabajos de investigación e implementación de TIC para atender problemas sobre la desigualdad económica y social. Asimismo, Walsham (2017) afirma que el estudio y la práctica de ICT4D son parte de una preocupación mayor del desarrollo a nivel mundial, aunque se han orientado básicamente a los países con mayor retraso socioeconómico y tecnológico. Este autor advierte también que es necesario situar la investigación de ICT4D en un contexto determinado, e identifica que, a diferencia de los estudios del área computacional enfocados

2 Este proyecto fue financiado por el Fondo Sectorial de Investigación para la Educación, SEP/CONACYT y la Universidad Autónoma de Aguascalientes, México. 
principalmente al diseño y aplicación de TIC para su posterior evaluación, los investigadores de las Ciencias Sociales se han preocupado por el análisis de grupos particulares de población y la apropiación de estas tecnologías.

Además de la línea de ICT4D, se encuentran otras que atienden el problema del acceso y apropiación de las TIC para el desarrollo y el cambio social desde diversas perspectivas, entre ellas las de adopción de innovaciones (Rogers, 1962), brecha digital (Selwyn, 2004), alfabetización tecnológica (Area, Gros \& Marzal, 2008) e inclusión digital (Tondeur, Sinnave, van Houtte \& van Braak, 2010; Warschauer 2002).

La Organización Iberoamericana de Juventud (OIJ), con base en la la Encuesta Iberoamericana de Juventudes, señala:

Tenemos frente a nosotros la evidencia que demuestra que la juventud vive hoy con mayor dramatismo el peso de la exclusión en el acceso a los servicios, las escasas oportunidades de empleo, los efectos de la violencia y una baja capacidad de incidencia en la política (OIJ, 2013, p. 13).

Por ello, el propósito de la revisión de literatura para integrar el presente estado de la cuestión fue identificar el empleo de las TIC para el desarrollo específico de los jóvenes, especialmente de quienes se encuentran en condiciones marginales o vulnerables en el difí- cil contexto actual (Reguillo, 2012). En los resultados de esta revisión, se describen los escasos trabajos localizados en el cruce entre ICT $4 D$ y desarrollo juvenil ${ }^{3}$. Estos estudios e intervenciones se presentan como referentes de futuras investigaciones y material de consulta para contribuir a una agenda y políticas públicas que mejoren las condiciones de este sector de la población.

En el primer apartado se presenta la estrategia que se siguió para estructurar el estado de la cuestión, desde la búsqueda de información, la selección de textos y su posterior clasificación. Los resultados de esta revisión se muestran en el segundo apartado, a través de una descripción cuantitativa del tipo de contenido que abordan los materiales localizados, y de acuerdo a los países en donde se llevaron a cabo y se publicaron. Después, se abre un bloque para el análisis de la información de los textos agrupados en esta revisión, la cual sin ser exhaustiva permite distinguir la discusión y crítica sobre cómo se han conceptualizado las tecnologías según su potencial para fomentar la capacidad de la población y mejorar sus condiciones de vida, centrándose por supuesto en la línea de estudio del desarrollo juvenil.

\section{Estrategia para la revisión de literatura}

La lógica de construcción del presente estado de la cuestión inició con una

3 Esta revisión de literatura se deriva de la investigación "Tecnologías de la Información y la Comunicación en Organizaciones Gubernamentales y Civiles para el Desarrollo de los Jóvenes”, la cual tiene como objetivo indagar cómo pueden integrarse las TIC de manera significativa en el quehacer de estas organizaciones. 
exploración previa entre los journals SAGE, considerando las siguientes palabras clave: Comunicación para el desarrollo o Communication for Development $(C 4 D)$, que es el concepto que prevalece en la literatura anglosajona. ${ }^{4}$ En esta primera revisión de literatura se encontró que la línea de Comunicación para el Desarrollo o $C 4 D$ es muy amplia y abarca varias estrategias, medios y TIC, por ello se decidió que sería más productivo y viable centrar la búsqueda en la línea de tecnologías para el desarrollo y el cambio social, Information and Technology Communication for Development (ICT4D).

Por otra parte, la línea Youth Development (YD) o Desarrollo Juvenil se identificó a nivel internacional para hacer luego el cruce con ICT4D a fin de seleccionar textos más específicos con estas mismas palabras clave, aunque también se incluyeron algunos relevantes sobre $C 4 D$ que explican la genealogía y vertientes de este campo.

La búsqueda de materiales publicados a partir del año 2000 se llevó a cabo finalmente en las siguientes bases de datos: SAGE, Ebsco, Scielo, E-book, Elsevier, Web of Science y Redalyc, así como en las memorias de los últimos congresos de la Asociación Latinoamericana de Investigadores de la Comunicación (ALAIC) y la
Asociación Mexicana de Investigadores de la Comunicación (AMIC). Se recopilaron un total de 100 textos, los cuales se analizaron y clasificaron en un archivo de Excel registrando la referencia completa, el abstract y los siguientes indicadores para tener una descripción general de las tendencias del campo: la línea de trabajo $(C 4 D$, ICT4D, YD u otras similares); el tipo de texto (teórico, metodológico, teórico/ metodológico, histórico, estado de la cuestión, reportes de investigación, investigación/intervención, análisis de políticas públicas y/o diagnósticos); el lugar donde fue realizado el estudio o la intervención, el país de adscripción institucional del autor(es), así como el de la publicación.

De los 100 textos revisados se seleccionaron 77, eliminando aquellos a los que no se tuvo acceso al artículo completo o bien, cuyo contenido no correspondía a la temática de interés. A pesar de que el cuerpo de estudio revisado fue amplio, es necesario reconocer que no fue exhaustivo, ya que además del campo académico existen estudios e intervenciones realizadas a partir de las prácticas de diversas organizaciones. Por otra parte, la revisión contempló solamente algunos bancos de datos, aunque se procuró que fueran entre los más reconocidos.

\footnotetext{
4 En América Latina ha prevalecido el concepto de Comunicación o Tecnologías para el Cambio Social, para distinguirse de las corrientes que comprenden el desarrollo como una propuesta unidireccional de los países que se consideran desarrollados en contraste con los subdesarrollados y que visualizan a la comunicación y a las TIC con un sentido instrumental para promover principalmente el desarrollo económico. Sin embargo, el concepto de desarrollo no necesariamente se limita a lo anterior como se argumenta más adelante en este artículo.
} 


\section{Descripción de estudios de ICT $4 D$ y} desarrollo juvenil

Un primer criterio para clasificar los 77 materiales que conformaron el estado de la cuestión fue la línea de estudio que abordaron: TIC para el desarrollo o ICT4D, Comunicación para el Desarrollo $(C 4 D)$, Desarrollo Juvenil o Youth Development $(Y D)$ y TIC o ICT desde otras perspectivas (Brecha digital, Alfabetización digital, Inclusión digital, Innovaciones tecnológicas, Movimientos sociales y Género, entre otras). Como se aprecia en la siguiente gráfica, una mayor cantidad corresponde a la línea ICT4D, y en particular los estudios sobre $Y D$ son los menos numerosos:

Gráfico 1. Líneas de estudio identificadas en 77 materiales (frecuencias)

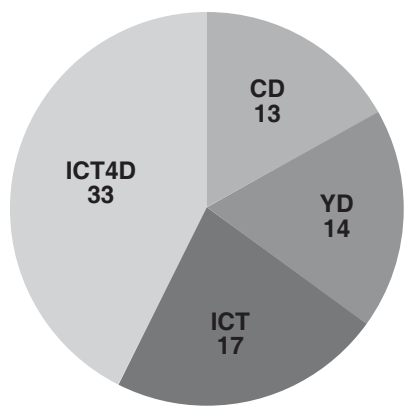

Fuente: Elaboración propia.

De acuerdo al contenido, en la tabla 1 se muestra el predominio de reportes de investigación sobre otro tipo de textos académicos. En cuanto a la región o país en el que se llevó a cabo el estudio y/o la intervención sobresalen las investigaciones comparativas entre países, aunque destacan aquellas realizadas en América Latina $y$ el Caribe, principalmente en Argentina (5), Colombia (4) y México (4). Estados Unidos es el país en el que se concentran estudios sobre Comunicación para el Desarrollo y en particular, es el que más ha contri- buido a la línea de Desarrollo Juvenil o Youth Development con 11 de los 14 trabajados publicados. En África se localizaron investigaciones en Sudáfrica, Kenia, Libia, Tanzania y Burundi; los países de Asia que han contribuido en estas líneas son la India, Israel, Corea del Sur, Camboya, Filipinas, Malasia e Indonesia. En estas dos últimas regiones se marca una tendencia por la línea general de ICT4D, y, por último, en Europa se identificaron investigaciones en España, Suecia y Bélgica. 
Tabla 1. Clasificación de trabajos por contenido y lugar de realización (frecuencias)

\begin{tabular}{|l|r|l|r|}
\hline \multicolumn{2}{|c|}{ Tipo de contenido } & \multicolumn{2}{c|}{ Regiones y países } \\
\hline Reportes de investigación & 32 & Varios países (estudios comparativos) & 24 \\
\hline Textos teóricos & 15 & América Latina y el Caribe & 22 \\
\hline Investigación / intervención & 8 & Estados Unidos & 12 \\
\hline Estados de la cuestión & 7 & África & 8 \\
\hline Análisis de políticas públicas & 7 & Asia & 8 \\
\hline Documentos teórico-metodológicos & 4 & Europa & 3 \\
\hline Diagnósticos & 2 & & \\
\hline Análisis desde perspectiva histórica & 0 & & 77 \\
\hline Total & 77 & Total & \\
\hline
\end{tabular}

Fuente: Elaboración propia.

A pesar de que una gran parte de los estudios se realizaron en América Latina y el Caribe, son otras las regiones y países de adscripción institucional de los autores, así como los lugares de las editoriales de las revistas o libros en los que se publicaron los textos revisados, considerando el país del editor en jefe. La información de la tabla siguiente muestra una tenden- cia a la coautoría y también son elevadas las publicaciones en coedición.

Estos datos muestran que se lleva a cabo un alto número de investigaciones empíricas en los países considerados en vías de desarrollo; sin embargo, la mayoría de los resultados de estos trabajos son publicados en países anglosajones como el Reino Unido y en Estados Unidos.

Tabla 2. Lugar de adscripción de autores y de editoriales de las publicaciones (frecuencias)

\begin{tabular}{|l|r|l|r|}
\hline \multicolumn{2}{|c|}{ País de adscripción de autores } & \multicolumn{2}{c|}{ País de editorial } \\
\hline Distintos países (coautorías) & 31 & Reino Unido & 27 \\
\hline Estados Unidos & 15 & Distintos países (coediciones) & 24 \\
\hline Reino Unido & 9 & Estados Unidos & 9 \\
\hline Argentina & 6 & Colombia & 7 \\
\hline Sudáfrica & 4 & México & 4 \\
\hline Canadá & 4 & Argentina & 3 \\
\hline México & 4 & Canadá & 3 \\
\hline Colombia & 4 & & \\
\hline Total & 77 & Total & 77 \\
\hline
\end{tabular}

Fuente: Elaboración propia. 
En algunos países considerados altamente desarrollados es donde se concentran no solo la publicación, sino la adscripción de los investigadores que tratan estos temas. Por lo tanto, el trabajo académico sobre estas líneas de estudio y el pensamiento en torno al desarrollo se da fuera de las regiones más vulnerables en donde sí se lleva a cabo la mayor parte de los estudios empíricos.

\section{Perspectivas críticas sobre TIC y de- sarrollo}

En la revisión de literatura sobre TIC para el desarrollo sobresale una crítica al concepto de desarrollo centrado sólo en lo económico y la manera en la cual se ha impulsado a través del incremento de la infraestructura, el acceso y la evaluación del impacto de estas tecnologías (Díaz \& Urquhart, 2012; Heeks, 2010; Leye, 2007; Mansell, 2014; Tribe, 2011).

Heeks (2010) señala que a pesar de que se han invertido grandes cantidades en infraestructura por parte de los organismos internacionales, países en desarrollo y los propios usuarios, no ha sido evidente la contribución de las TIC al mejoramiento de la población global, es decir, ¿̇uál ha sido la contribución de este gasto al desarrollo?

En este sentido, el pensamiento de Sen (2000) se ha asumido como una alternativa a la perspectiva económica para comprender la promoción del desarrollo, no de una manera homogénea, sino a partir de estrategias situadas en cada contexto y como puente entre la visión de los organismos internacionales y la academia. Este autor define el desarrollo como la necesidad de superar la persistencia de la pobreza y las necesidades básicas a través de reconocer el papel que desempeñan los diferentes tipos de libertad para trascender las carencias. Distingue entre cinco tipos de libertad: las libertades políticas, los servicios económicos, las oportunidades sociales, las garantías de transparencia y la seguridad protectora.

Según este enfoque, la expansión de la libertad es tanto el fin primordial del desarrollo como su medio principal. El desarrollo consiste en la eliminación de algunos tipos de falta de libertad que dejan a los individuos pocas opciones y escasas oportunidades para ejercer su agencia razonada. La eliminación de la falta de libertades fundamentales -es lo que sostenemos aquí- es parte constitutiva del desarrollo (Sen, 2000, p. 16).

El enfoque sociocultural del estudio de las TIC coincide con el pensamiento de Sen (2000) al sostener que para lograr el cambio social es necesario centrarse en el contexto cultural del desarrollo humano y no sólo en las tecnologías o en lo económico.

El enfoque sociocultural aporta un sistema teórico para la explicación del proceso de construcción de conocimientos, considerando las TIC no como meros soportes técnicos, sino como nuevos lenguajes para el entendimiento humano, para compartir experiencias y conocimientos. En este sentido, las TIC son herramientas culturales, nuevos espacios estratégicos de las mediaciones sociales, que posibilitan procesos de interacción social y 
reconstrucción cultural (Dubois y Cortés, 2005, p. 29).

Esto implica un viraje del paradigma tecnológico-informacional hacia el del reconocimiento del sujeto. Por lo tanto, resulta pertinente una teoría que parta de las percepciones y subjetividad de las personas, que capte la comunicación y el desarrollo como procesos dialogantes que se desprendan de la intersubjetividad. Esta visión plantea el conocimiento como un Bien Público Global (BPG). En consecuencia, la política de expansión de las TIC se conceptualiza como un instrumento al servicio de ese objetivo. Es decir, no se trata de afirmar a priori las posibilidades de las tecnologías sino analizar, desde la perspectiva de los usuarios, cuándo y cómo su empleo se encamina realmente a una mejora del desarrollo humano considerando no sólo la capacidad económica sino además las capacidades humanas y morales. "La pregunta clave es: ¿de qué manera encontramos formas de aplicar el enfoque de las capacidades como base de un modelo para la distribución justa y el uso éticamente beneficioso de las TIC en un mundo globalizado?" (Dubois y Cortés, 2005, p. 14).

Varios autores confluyen en torno a esta pregunta con propuestas, en su mayoría basadas en el pensamiento de Sen (2000), para integrar una conceptualización de desarrollo más amplia en la investigación e intervención. Estos planteamientos son interesantes debido a que aportan elementos clave para nutrir el diseño de futuros estudios y prácticas, por ello aquí se describen algunos de los principales.
Heffernan, Et. al., (2016), sostienen que existen diversos marcos teóricos que orientan la investigación y la práctica de $I C T 4 D$, por ello resulta fundamental definir teóricamente los conceptos de desarrollo, comunicación y distribución para crear un marco común que fundamente y relacione la teoría con la práctica. Argumentan que las micro-narrativas sobre desarrollo son más pertinentes que las grandes narrativas que presentan problemas para ser aplicadas a proyectos y prácticas. Mithoko \& Pade-Khene (2013) coinciden que ante la creciente variedad de perspectivas y enfoques en ICT4D es necesario orientar la investigación y la práctica con base en la sistematización de las bases éticas del desarrollo y las TIC. La práctica ética debe considerar la colaboración y participación, el contexto socioeconómico, los costos y los intereses de los actores y sectores involucrados. A su vez, Thakur (2012) subraya la necesidad de tener claridad sobre cómo se entiende el desarrollo, las TIC y el propósito para el cual se aplican, sea de intervención, para ofrecer soluciones prácticas, con fines del mercado y la empresa; para políticas públicas o generar nuevo conocimiento; asimismo falta mayor confluencia y colaboración entre estos campos.

Andrade \& Urquhart (2010) proponen la teoría del actor-red para analizar la confluencia o no entre la red social de una comunidad y la que se establece con la implementación de las TIC para articular sociedad y tecnología. A nivel comunitario, Breytenbach, De Villiers \& Jordaan (2013), con base en 
experiencias de intervención, ofrecen modelos para lograr proyectos maduros y sustentables de ICT4D que aceleren el desarrollo. Además, Loh (2013) revisa tanto las vulnerabilidades de una comunidad como sus capacidades para comprender mejor cómo aportar a su desarrollo integral.

Kleine (2010) realizó una propuesta para operacionalizar la perspectiva de Sen (2000). Argumenta que el problema metodológico que se presenta es que si se clasifican y operacionalizan las capacidades en cierta manera se determinan y no se permite abrir los estudios a las diversas posibilidades de elección que pudieran asumir los actores sociales ante las TIC. Para ello, propone un "marco de elección" con base en el trabajo etnográfico que realizó en Chile y en las teorías sobre empoderamiento (empowerment framework), y vidas sustentables (sustainable livelihoods). En este sentido, Johri \& Pal (2012) proponen un marco con un diseño amigable de TIC para el desarrollo humano.

La conceptualización más compleja de desarrollo ha desafiado no sólo las metodologías para implementar proyectos en esta línea, sino la manera en la cual se evalúan. Heeks (2010) argumenta que se requiere una perspectiva interdisciplinar que integre el desarrollo económico y el logro de una vida sustentable y libre. Para ello, propone un modelo integral de "cadena de valor", para analizar las contribuciones de las TIC al desarrollo, en cuanto a cómo su implementación se traduce en infraestructura y políti- cas públicas, acceso, apropiación e impacto.

\section{Resultados y discusiones en torno a una agenda de estudios sobre TIC, de- sarrollo y jóvenes}

Este apartado describe y discute los resultados de esta revisión de literatura. Primeramente, aborda las tendencias encontradas en estudios dirigidos a comunidades marginadas, los cuales predominan, para posteriormente dar cuenta de los escasos estudios que se orientan de manera específica a los jóvenes.

\subsection{El desarrollo en comunidades marginadas}

La mayoría de los estudios y proyectos de intervención en la línea de ICT4D se centran en comunidades marginadas. Una clara tendencia es la postura epistemológica de integrar diversas perspectivas, no sólo la académica, en las búsquedas por generar alternativas en el papel de las TIC en la promoción del desarrollo. Pimienta y Barnola (2005) proponen generar comunidades virtuales y metodologías en línea para reflexionar, discutir e informarse colectivamente sobre el papel de las TIC entre investigadores y activistas. En esta tendencia, la metodología del "laboratorio viviente" promueve la colaboración entre "sector público, privado y civil en la cual estos actores como socios co-crean nuevos productos, servicios, empresas en ambientes de la vida real y en redes virtuales en esferas multicontextuales" (Pade-Khene, Palmer \& Kavhai, 2010, p. 266). 
Otra propuesta, es cambiar la conceptualización de las poblaciones marginadas. Rangaswamy \& Nair (2012) señalan la importancia de reconocer la capacidad de los pobres para ser agentes activos y productores innovadores de productos y servicios en torno a las TIC y no comprenderlos como meros consumidores pasivos. Salinas, Huerta, Porras, Amador y Ramos (2006) apoyan la tendencia de asumir la centralidad del usuario al proponer un modelo de alfabetización digital para emplear las TIC de manera significativa, a la par del desarrollo de proyectos relevantes para sus vidas. Incluso, Carniglia (2014) argumenta que la apropiación de las tecnologías info-comunicacionales trasciende la perspectiva mercantilista y del desarrollo económico hacia un modelo de servicio público para un concepto de ciudadanía ampliada, en particular para la población rural. Asimismo, se ha señalado que las TIC para el desarrollo son importantes para lograr una cultura del servicio, es decir, que se insertan en los derechos humanos universales (White, 2004).

Sin duda alguna, los programas para el desarrollo son necesarios entre la población que carece de poder económico, político y cultural y no tienen acceso a los servicios básicos, sin embargo, White (2004) argumenta que la perspectiva de las TIC para el desarrollo no debe enfatizar sólo la marginalidad. Para este autor el problema más grave es que no se les reconoce a estos sectores de la población su capacidad para contribuir a la comunidad y por el contrario se les estigmatiza. Por ello, un tema clave es resignificar y empoderarlos desde el género, la diversidad sexual, religiosa y racial. Walsham (2017) coincide al cuestionar los problemas que se han atendido a través de las TIC, señala que ha sobresalido la promoción de la economía y la erradicación de la pobreza, sin embargo, es necesario reconocer otros. A esta diversidad de perspectivas se puede añadir la distinción entre la población por edad, esto resulta particularmente relevante en el caso de los jóvenes, dado que son frecuentemente estereotipados o criminalizados.

\subsection{TIC para el desarrollo y jóvenes. Un cruce poco atendido}

Los problemas que enfrentan los jóvenes han sido abordados por una línea de investigación e intervención nombrada Youth Development, o Desarrollo Juvenil. Esta perspectiva surgió a partir de una crítica hacia las intervenciones dirigidas a jóvenes que sólo respondían a situaciones de crisis con poca atención a la prevención, ampliando este marco hacia el estudio longitudinal que identifica múltiples e interrelacionados elementos predictivos del comportamiento juvenil problemático, como factores de protección que contribuyan a un desarrollo juvenil saludable (Catalano, Berglund, Ryan, Lonczak \& Hawkins, 2004; Lynn \& Terling, 2014). Varios estudios confluyen en esta perspectiva (Borden, Perkins, Villaruel, CarletonHug, Stone \& Keith, 2006; Christens \& Dolan, 2011; Furlong \& Cartmel, 2007; Sy, Greaney, Nigg \& Hirose-Wong, 2015; Zeldin, Camino \& Calvert, 2007). 
Huebner, Walker \& McFarland (2003) señalan que en el trabajo a favor de los jóvenes se requieren dos asuntos clave: primero, el desarrollo de un marco para comprender los fundamentos y la articulación de lo que es el desarrollo de los jóvenes; y segundo, sobre el perfil de quienes atienden a los jóvenes y las metodologías, procesos efectivos y estrategias para apoyarlos. Algunos estudios que se centran en analizar el perfil de quienes trabajan con los jóvenes son los de Krauss, Idris, Tamam, Suandi, Ismail, Bandar \& Dahalan, 2012; y Walker, 2011.

Precisamente, las TIC constituyen una fuente importante de recursos para promover a los jóvenes. En esta revisión se encontró que la mayoría de los estudios o intervenciones sobre TIC para el desarrollo se aplicaron a poblaciones marginadas o vulnerables y muy escasos estudios o intervenciones se enfocaron de manera específica al desarrollo de los jóvenes, parece ser un cruce poco atendido ${ }^{5}$.

Entre estos escasos estudios, London, Pastor, Servon, Rosner \& Wallace (2010) analizaron de qué manera los centros de acceso a TIC son capaces de promover el desarrollo de jóvenes. Los hallazgos mostraron que el acceso y uso de la tecnología está estrechamente vinculado a un desarrollo positivo de la juventud. Señalan que generalmente el acceso y uso de una computadora e internet se relacionan solo con una mejora académica y laboral y menos con el impac- to más amplio que pueden tener entre los jóvenes, como la construcción de habilidades tecnológicas, de relaciones sociales, la promoción de su voz y de su compromiso cívico; por ello recomiendan:

Fusionar la literatura sobre tecnología para comunidades con el desarrollo para jóvenes para generar un marco que apoyara a los profesionales de la tecnología y a los operadores de programas a comprender el poder de la tecnología para promover resultados en la juventud, que aparentemente no están relacionados con la tecnología en sí misma (London, Et. al., 2010, p. 201).

Por su parte, estudios e intervenciones como la de Badillo (2011), se orientó a generar una estrategia de comunicación y educación ambiental a través de las TIC para detonar el discurso y fomentar el compromiso de los jóvenes sobre el desarrollo sostenible. A su vez, Ville (2016) revisó el potencial de las aplicaciones en móviles entre jóvenes agricultores.

Además de los estudios anteriores que exploran el potencial de las TIC para desarrollar a los jóvenes en distintos ámbitos, se identificó un trabajo en donde los jóvenes logran desarrollar su potencial al promover el desarrollo de comunidades a través de las TIC (Mihyo \& Ogbu, 2000).

Otros trabajos se abocan a problemáticas específicas como el de Eglinton, Gubrium \& Wexler (2017) que emplearon

45 La revisión de trabajos que cruzan el estudio del Desarrollo Juvenil con ICT4D fue del 2000 al 2017. 
la metodología de la digital storytelling para enfrentar altos niveles de suicidio y consumo de drogas, mediante una plataforma en la cual los jóvenes se representan a ellos mismos, su cultura e identidad para enfatizar aspectos positivos de sus vidas y relaciones con personas que son importantes para ellos. Hanckel (2016) se centró en jóvenes discriminados por su preferencia sexual e intervino a partir de una web-app integrada para proporcionar información, servicios y narrativas visuales sobre problemas de identidad y sexualidad. El trabajo de Yarmuth, Patterson, Burton, Douglas, Taylor \& Boyle (2012) abordó la problemática de la violencia de pareja entre jóvenes al revisar los hábitos de este grupo de edad, entre ellos su consumo de medios y TIC para determinar estrategias de comunicación pertinentes. Estos autores reportan que problemas como adicciones, desórdenes de conducta, delincuencia y comportamiento antisocial, fracaso académico y embarazo juvenil también pueden ser atendidos a través de intervenciones basadas en la comunicación y las TIC. Otro estudio sobre violencia fue el de Mareschal, McKee, Jackson \& Hanson (2007), quienes crearon un programa innovador para prevenir y reducir la violencia juvenil. El programa consistió en la aplicación de una red de servicios en línea que promueve la comunicación y la toma de decisiones para la resolución de conflictos y la solución de problemas de manera colaborativa.

Finalmente, se identificaron un par de estudios que exploraron la contribu- ción del uso de TIC para apoyar jóvenes con discapacidades (Näslund \& Gardelli, 2013; y Schreuer, Keter \& Sachs, 2014).

En esta revisión de literatura se encontraron escasamente 10 trabajos en la línea de TIC para el desarrollo aplicados al contexto y necesidades de los jóvenes, por lo tanto, es un cruce de temas poco atendido.

\section{Conclusiones: Aportes para una agenda de estudios sobre TIC, desa- rrollo y jóvenes}

Walsham (2017) señala que las primeras iniciativas de ICT $4 D$ han perdido fuerza, y el campo requiere actualizar su pertinencia para recuperar su legitimidad. En este sentido propone dirigir la aplicación de las TIC a sectores de la población más específicos, como el caso de los jóvenes, lo cual puede responder a la necesidad de comprender mejor al usuario y en consecuencia lograr una apropiación más significativa y con resultados más evidentes.

La presente revisión de literatura se concentró sólo en la producción académica, sin embargo, la línea de estudio de TIC para el desarrollo o ICT4D no se limita a lo académico, sino que constituye un vasto campo interdisciplinario que abarca políticas públicas, diagnósticos, proyectos, intervenciones y prácticas. Además, se complejiza debido a la diversidad de disciplinas que abordan las TIC y de perspectivas epistemológicas, teóricas y metodológicas que se proponen. Incluso, resulta difícil precisar límites o distinciones entre líneas de estudio similares. Revisando la 
rica historia de teorías y visiones que nutren los estudios y prácticas actuales, difícilmente pueden comprenderse de manera lineal, en el sentido de un avance histórico en donde los aportes del pasado se van superando. El asunto es más complejo debido a que las diversas propuestas deben valorarse de acuerdo a su momento histórico, contexto, alcances y limitaciones para reconocer que actualmente, de acuerdo a cada caso de estudio o intervención, se puede revisar todo el bagaje anterior sin desconocerlo de antemano. Indudablemente, una agenda pertinente a futuro sería continuar examinando la genealogía y trayectoria de las disciplinas, campos y prácticas que se centran en impulsar el desarrollo a través de las TIC para hacer un análisis transversal y así optimizar los logros de cada una de estas perspectivas.

A pesar de las limitaciones de esta revisión, se señalan aportes concretos para nutrir el trabajo de investigación e intervenciones. Claramente una tendencia central es la discusión sobre cómo se ha comprendido el concepto de desarrollo, lo cual orienta el sentido que adquiere el quehacer de las TIC en este marco. No solo la academia, sino además los organismos internacionales, las políticas públicas y la práctica deben partir de hacer explícita la base conceptual e incluso ética de desarrollo que asumen. Aunque persiste una comprensión del desarrollo basada en la visión de los países hegemónicos, en la modernidad y en una concepción técnico-instrumental, se confluye hacia un entendimiento del desarrollo en un sentido integral y más complejo, incorporando varios aspectos del desarrollo humano y fundamentalmente tomando en cuenta la perspectiva y los deseos de los actores sociales que se pretenden promover. Ahora las tendencias y las discusiones se centran en cómo incorporar esto en las prácticas de investigación e intervenciones para evaluar su pertinencia e impacto en esta lógica. Un segundo eje clave de la discusión es avanzar sobre el papel de las TIC en el desarrollo en varios niveles, desde el global, en países, organizaciones, comunidades y en lo individual. En cuanto a la presencia de esta línea de estudio y práctica es importante señalar las hegemonías de algunos países, principalmente en lo referente a la capacidad de realizar estudios de amplio alcance y en la publicación de resultados de investigación. La generación de conocimiento es innegablemente un trabajo que se enriquece si se estimula una mayor colaboración entre varios sectores y desde las perspectivas de diversos lugares en el mundo.

El propósito central de realizar esta revisión de literatura fue identificar cómo se han orientado los esfuerzos de la investigación e intervenciones con TIC al caso de los jóvenes. Prevaleció el trabajar en comunidades marginadas, en pobreza, y si se revisan los actuales objetivos de desarrollo sostenible también destaca el caso de las mujeres. Los jóvenes y los niños son considerados principalmente desde la perspectiva de emplear las TIC para su educación o sobre el generar políticas y estrategias para garantizar su seguridad en el contexto 
de la sociedad de la información. Por otra parte, se suele asumir que debido a que los jóvenes son el sector de la población que hace uso de las TIC no resulta necesario atender las desigualdades que existen entre ellos mismos en cuanto a su uso para su inclusión social. No se ha considerado de manera suficiente el potencial de las TIC para enfrentar y solu- cionar la creciente problemática de los jóvenes a nivel mundial. Se concluye que resulta pertinente continuar explorando cómo aprovechar las tecnologías para promover el desarrollo humano e integral, lo cual en cruce con la línea de trabajo para el Desarrollo Juvenil abre una nueva agenda no solo necesaria, sino además desafiante.

\section{Bibliografía}

Andrade, A. \& Urquhart, C. (2010). The affordances of actor network theory in ICT for development research. Information Technology \& People, 23(4), pp. 352-374. doi: https://doi. org/10.1108/09593841011087806.

Area, M., Gros, B. \& Marzal, M. (2008). Alfabetizaciones y tecnologías de la información y la comunicación. España: Editorial Síntesis.

Badillo, M. E. (2011). Estrategia de comunicación y educación mediada por TIC para el fomento del desarrollo sostenible en cinco colegios de Palmira. Entramado, 7(1), pp. 128-145.

Baelden, D. \& Audenhove, L. (2015). Participative ICT4D and living lab research: The case study of a mobile social media application in a rural Tanzanian University setting. Telematics and Informatics, 32(4), pp. 842-852. doi: http://dx.doi.org/10.1016/j.tele.2015.04.012.

Borden, L., Perkins, D., Villaruel, F., Carleton-Hug, A., Stone, M. \& Keith, J. (2006). Challenges and opportunities to latino youth development programs. Hispanic Journal of Behavioral Sciences, 28(2), pp. 187-207. doi: http://doi.org/10.1177/0739986306286711.

Breytenbach, J., De Villiers, C. y Jordaan, M. (2013). Communities in control of their own integrated technology development processes. Information Technology for Development, 19(2), pp. 133-150. doi: https://doi.org/10.1080/02681102.2012.714348.

Carniglia, E. L. (agosto de 2014). Comunicación para el desarrollo como derecho humano: el acceso a las TIC en el mundo rural. En C. Bolaño (Presidencia). Comunicación, tecnología y desarrollo. Simposio llevado a cabo en el XII Congreso Latinoamericano de Investigadores de la Comunicación ALAIC 2014, Lima, Perú.

Catalano, R. F., Berglund, M. L., Ryan, J. A., Lonczak, H. S. \& Hawkins, J. D. (2004). Positive youth development in the United States: Research findings on evaluations of positive youth development programs. The ANNALS of the American Academy of Political and Social Science, 591(1), pp. 98-124. doi: https://doi.org/10.1177/0002716203260102.

Christens, B. \& Dolan, T. (2011). Interweaving youth development, community development, and Social change through youth organizing. Youth \& Society, 43(2), pp. 528-548. doi: https://doi.org/10.1177/0044118X10383647.

Díaz, A. \& Urquhart, C. (2012). Unveiling the modernity bias: a critical examination of the politics of ICT4D. Information Technology of Development, 18(4), pp. 281-292. https://doi.org /10.1080/02681102.2011.643204. 
Dubois, A. y Cortés, J. J. (2005). Nuevas tecnologías de la comunicación para el desarrollo humano. Bilbao: Hegoa.

Eglinton, K., Gubrium, A., \& Wexler, L. (2017). Digital storytelling as arts-inspired for engaging, understanding, and supporting indigenous youth. International Journal of Education \& the Arts, 18(5), pp. 2- 29.

Furlong, A. \& Cartmel, F. (2007). Young People and Social Change. New Perspectives. London: Open University Press.

Hanckel, B. (2016). Mitigating risk and facilitating access to capabilities: the role of affect in the design of an ICT- tool for queer youth in Asia, Emotion, Space \& Society 18, pp. 35-43. doi: https://doi.org/10.1016/j.emospa.2016.01.007

Heeks, R. (2010). Do information and communication technologies (ICTs) contribute to development? Journal of International Development, 22, pp. 625-640. doi: https://doi. org/10.1002/jid.1716

Heffernan, C., Lin, Y., and Kim, T. (2016) Drawing from Development: Towards Unifying Theory and Practice of ICT4D. Journal of International Development, 28, pp. 902-918. doi: http://doi.org/10.1002/jid.2882.

Huebner, Walker, J. \& McFarland, M. (2003). Staff development for the youth development professionalm: A critical framework for understanding the work. Youth Society, 35(2), pp. 204-225. doi: https://doi.org/10.1177/0044118X03255024.

Johri, A. \& Pal, J. (2012). Capable and convivial design (CCD): a framework for designing information and communication technologies for human development. Information Technology for Development, 18(1), 61-75. doi: https://doi.org/10.1080/02681 102.2011.64320 2.

Kleine, D. (2010). ICT4 WHAT? -Using the choice framework to operationalise the capability approach to development. Journal of International Development, 22(5), pp. 674-692. doi: https://doi.org/10.1109/ICTD.2009.5426717

Krauss, S., Idris, K. Tamam, E., Suandi, T., Ismail, I., Bandar, N. \& Dahalan, D. (2012). Exploring professionalism among youth work practitioners in Malaysia: A measurement development study. Young, 20(3), pp. 297-322. doi: https://doi. org/10.1177/110330881202000305

Leye, V. (2007). UNESCO, ICT corporations and the passion of ICT for development: modernization resurrected. Media Culture Society, 29(6), pp. 972-993. doi: https://doi. org/10.1177/0163443707081711

Loh, Y. (2013). Approaches to ICT for development (ICT4D): Vulnerabilities vs. capabilities. Information Development, 31 (3),pp. 229-238. doi: https://doi.org/10.1177/0266666913513198

London, R., Pastor, M., Servon, L., Rosner, R. \& Wallace, A. (2010). The role of community technology centers in promoting youth development. Youth Society, 42(2), pp. 199-228. doi: https://doi.org/10.1177/0044118X09351278

Lynn, C. \& Terling, T. (2014). Exploring the impact of a wilderness-based positive youth development program for urban youth. Journal of experimental education, 37(4), pp. 335350. doi: https://doi.org/10.1177/1053825913503113 
Mansell, R. (2014). Power and interests in information and communication and development: exogenous and endogenous discourses in contention. Journal of International Development, 26, pp. 109-127. doi: http://doi.org/10.1002/jid.1805

Mareschal, P., McKee, W., Jackson, E. \& Hanson, K. (2007). Technology-based approaches to preventing youth violence: a formative evaluation of program development and implementation in four communities. Youth Violence and Juvenile Justice, 5, pp.168-187. doi: https://doi.org/10.1177/1541204006295148

Mihyo, P. \& Ogbu, O. (2000). A youth leadership program for Africa. En O. Ogbu, O. \& P. Mihyo (Eds.), African youth on the information highway. Participation and leadership in community development (pp. 1-22). Ottawa: International Development Research Centre.

Mithoko, H. y Pade-Khene, C. (2013). Towards a theoretical framework on ethical practice in ICT4D programmes. Information Development, 29(1), pp. 36-53. doi: http://doi. org/10.1177/0266666912449456

Näslund, R. \& Gardelli, A. (2013). 'I know, I can, I will try': youths and adults with intellectual disabilities in Sweden using information and communication technology in their everyday life. Disability E Society, 18(1), pp. 28-40. doi: http://dx.doi.org/10.10801/09687599. 2012.695528

Organización Iberoamericana de Juventud (OIJ) (2013). $1^{a}$ Encuesta Iberoamericana de Juventudes. Informe Ejecutivo. Madrid: PNUD/CEPAL/UNAM.

Pade-Khene, C. Palmer, R. \& Kavhai, M. (2010). A baseline study of a Dwesa rural community for the Siyakhula information and communication technology for development project: understanding the reality on the ground. Information Development, 26(4), pp. 265-288. doi: https://doi.org/10.1177/0266666910385374

Pimienta, D. y Barnola, L. (2005). En la búsqueda colectiva de un impacto social positivo a la Internet Latinoamericana. La experiencia del proyecto sobre metodología e impacto social de las tecnologías de la información y de la comunicación en América Latina y el Caribe (MISTICA) y la constitución de la red de observación OLISTIC. Santiago de Compostela: Universidad de Santiago de Compostela.

Rangaswamy, N. \& Nair, S. (2012). The PC in an Indian urban slum: enterprise and entrepreneurship in ICT4D 2.0. Information Technology for Development, 18(2), pp. 163-180. doi: https://doi.org/10.1080/02681102.2011.643211

Reguillo, R. (2012). Culturas juveniles. Formas políticas del desencanto. Buenos Aires: Siglo Veintiuno Editores.

Rogers, E. (1962). Diffusion of innovations. New York: Free Press.

Salinas, B., Huerta, M. G., Porras, I. H., Amador, S. E. y Ramos, J. M. (2006). Uso significativo de la tecnología en la educación de adultos en el medio rural: Resultados de la aplicación piloto de un modelo. Revista Mexicana de Investigación Educativa, 11(28), pp. 31-60.

Schreuer, N., Keter, A. \& Sachs, D. (2014). Accessibility to information and communications technology for the social participation of youths with disabilities: A two-way street. Behavioral Sciences E The Law, 32(1), pp.76-93. doi: http://doi.org/10.1002/bsl.2104

Selwyn, N. (2004). Reconsidering political and popular understandings of the digital divide. 
New Media \& Society, 6(3), pp. 341-362. doi: https://doi.org/10.1177/1461444804042519 Sen, A. (2000). Desarrollo y libertad. Barcelona: Planeta.

Sy, A., Greaney, M., Nigg, C. \& Hirose-Wong, S. (2015). Developing a measure to evaluate a positive youth development program for native hawaiians. Asia Pacific Journal Public Health, 27(2), pp. 114-162. doi: https://doi.org/10.1177/1010539511427760

Thakur, D. (2012). Leveraging information and communication technologies for development (ICTD) in the Caribbean. Geography Compass, 6(1), pp. 1-18. doi: https://doi. org/10.1111/j.1749-8198.2011.00467.x

Tondeur, J. Sinnaeve, I, van Houtte, M. \& van Braak, J. (2010). ICT as cultural capital: The relationship between socioeconomic status and the computer-use profile of young people. New Media \& Society, 13(1), pp. 151-168. doi: https://doi.org/10.1177/1461444810369245

Tribe, L. (2011). Forgettin the "C" in ICT4D: A critical examination of the global alliance for ICT and development. (Unedited Master's thesis). Carleton University, Ottawa, Ontario, Canada.

Ville, M. (2016). Mobile applications, solution for sustainable agriculture? Study of mAgriculture services in Kenya. (Unedited Master's thesis). Lappeenranta University of Technology, Lappeenranta, Finlandia.

Walker, K. (2011). The multiple roles that youth development program leaders adopt with youth. Youth Society, 43(2), pp. 635-655. doi: https://doi.org/10.1177/0044118X10364346

Walsham, G. (2017) ICT4D research: reflections on history and future agenda. Information Technology for Development, 23(1), pp. 18-41. doi: https://doi.org/10.1080/02681102.2016.12 46406

Warschauer, M. (2002). Reconceptualizing the digital divide. First Monday, Peer-reviewed. Journal on the Internet, 7(7). Recuperado de: http://firstmonday.org/article/view/967/888

White, R. (2004). Is 'empowerment' the answer? Current theory and research on development communication. Gazette, The International Journal for Communication Studies, 66(1), pp. 7-24. doi: https://doi.org/10.1177/0016549204039939

World Summit on the Information Society (WSIS), (2017). Recuperado de https:/www.itu. int/net4/wsis/forum/2017/

Yarmuth, M., Patterson, J., Burton, T., Douglas, C., Taylor, T. \& Boyle, M. (2012). Using research to understand youth in high-risk urban communities. Social Marketing Quarterly, 18(3), pp.187-202. doi: 10-1177/1524500412460668

Zeldin, S., Camino, L. \& Calvert, M. (2007). Toward an understanding of youth in community governance: Policy priorities and research directions. Análise Psicológica, 25(1), pp.77-95. 